\title{
D oenças crônicas não-transmissíveis no Brasil: um desafio para a complexa tarefa da vigilância
}

\author{
Chronic non-communicable diseases in Brazil: \\ a challenge for the complex task of surveillance
}

Ines Lessa 1

1 Instituto de Saúde Coletiva da Universidade Federal da Bahia. Rua Padre Feijó 29/40 andar, 40170-000, Salvador BA. inlessa@ufba.br

\begin{abstract}
There is a scarcity of literature in the field of public health with regard to the surveillance of non-communicable diseases but there are various proposals for programs and various networks in operation throughout the 5 continents. The subject is in debate all over the world not only because cardiovascular disease is now the leading cause of death in most countries, but because of what is already being considered a pandemic of obesity, including children and adolescents. The diabetes endemic is expected. In Brazil the situation is serious. The population is aging fast and this rapidly accentuates the importance of obesity and diabetes as a public health issue. H ealthcare up to now has been curative. However, this does not de facto and de jure assure the uninterrupted treatment of NCD within the basic healthcare system. The text presented gives a summary of the NCD surveillance in general and comments on theSTEPwise approach to NCD surveillance proposed by theWorld Health Organization for the emerging countries as Brazil. To have this proposal accepted, a critical analysis of the secondary data available in Brazil and mentioned in the proposal is necessary. M ethodologically standardized primary data with an emphasis on risk factors should also be produced within a short timeframe.

Key words Non-communicable diseases, Surveillance, Programs, Pandemic, Epidemic
\end{abstract}

Resumo A literatura na área da saúde pública é pobre em textos sobre vigilância epidemiológica (VE) para DCNT, mas contém várias propostas de programas e várias redes em andamento nos 5 continentes. 0 tema encontra-se em destaque mundial, não só pela liderança das doenças cardiovasculares na mortalidade na grande maioria dos países, como pela já considerada pandemia da obesidade, inclusive em crianças e adolescentes. A endemia do diabetes é esperada. No Brasil a situação é grave. 0 envelhecimento populacional é rápido, com expectativa de aumento real das freqüências da obesidade e o diabetes como problema populacional sem concomitantes estratégias de proteção e promoção da saúde no contexto das DCNT. Predomina maciçamente a prática clínica, mas sem assegurar o tratamento ininterrupto para as DCNT na rede básica de saúde. 0 texto apresentado aborda sumariamente a VE para DCNT de modo geral e comenta o M étodo Progressivo de VE para DCNT proposto pela OMS, no caso do Brasil. Para aceitá-la é imprescindível uma análise crítica dos dados secundários nacionais disponíveis e mencionados na proposta. É também necessário produzir, a curto prazo, dados primários metodologicamente padronizados com enfoque para fatores de risco.

Palavras-chave Doenças crônicas não-transmissíveis, Vigilância, Programas, Pandemia, Epidemia 


\section{Introdução}

Os conhecimentos sobre a epidemiologia das doenças crônicas não-transmissíveis (DCNT), tratamentos, metodologias para estudos de tendências originaram-se nos países desenvolvidos da América do Norte e Europa. Também deles procedem os conhecimentos sobre prevenção e controle, mas os resultados nem sempre têm sido favoráveis. As epidemias de fatores de risco como a da obesidade e do diabetes surgiram nos últimos anos, neles começaram e agora se disseminam por outros países (Jacoby, 2004; Eyre et al., 2004; Stein \& Colditz, 2004). A vigilância epidemiológica para as DCNT nos Estados Unidos iniciou-se em torno de 1923 e acelerou-se a partir dos anos 70, por metodologias mais modernas. Por diversas razões os países em desenvolvimento estão reproduzindo, de modo muito acelerado, a história das DCNT dos países desenvolvidos (Reddy \& Yusuf, 1998) com um agravante: a maior parte da população de quase todos eles convive com a pobreza e com imensas desigualdades sociais. No caso do Brasil as dificuldades em lidar com essas doenças têm um desafio a mais: a dimensão continental do País para implementar programas abrangentes para DCNT.

A literatura na área da saúde pública é pobre em textos sobre vigilância epidemiológica (VE) para DCNT, mas contém muitas propostas de programas e vários em andamento. 0 tema encontra-se em destaque no momento, tendo em vista as perspectivas da disseminação das epidemias desse tipo de doença, resultando em endemias e os custos sociais advindos.

Neste texto pretende-se abordar sumariamente a questão das DCNT e aVE para DCNT de modo geral e comentar a adaptação da proposta do "STEP wise approach for surveillance of non-communicable disease" (WH O, 2001) para o Brasil.

A partir dos últimos anos da década de 1990 e neste início do século 21, a prevenção para DCNT, em especial das cardiovasculares, vem sendo preocupação de várias organizações internacionais, enfatizando os chamados países do Terceiro M undo (W H O, 2002, 2003; Nissinen et al., 2001). Bilhões de pessoas estão vivendo mais, enquanto as doenças cardiovasculares parecem novamente em ascensão (M itka, 2004). A identificação da pandemia das doenças cardiovasculares (Reddy \& Yusuf, 1998; M agnus \& Beaglehole, 2001; Beaglehole \& Yach, 2003) em torno de 1997 foi a primeira de uma série de outras esperadas. Seguiram-se as epidemias de sobrepeso e obesidade nos países desenvolvidos, inclusive a detectada elevação das suas prevalências entre crianças e adolescentes, com registros da ocorrência do diabetes tipo 2 nesses grupos etários ( $M$ oakdad et al., 2001; Eyre et al., 2004). Essa epidemia já ocorre em vários dos países emergentes, com perspectivas reais de pandemia. Epidemias e pandemias de DCNT, associadas à crescente longevidade da população de países emergentes, determinam graves conseqüências sociais, pois são países despreparados para deter a carga de doença que prevalecerá nas próximas décadas (Yach et al., 2004).

Não sem razão, entre os modismos da literatura médico-social da atualidade encontramse, por um lado, os determinantes comportamentais e os político-econômicos e sociais para as DCNT, destacando-se entre eles: síndrome plurimetabólica, inflamação, mensurada pela Proteína $\mathrm{C}$ reativa de alta sensibilidade (seu marcador mais potente), aterosclerose, exposição a fatores de risco nutricionais desde a vida intra-uterina ou nos primeiros anos de vida, carga de doença, "globalização" e as iniqüidades sociais (M urray \& Lopez, 1996; Yusuf et al., 2001; Grundy, 2003). Em grande parte desses fatores existe uma intensa inter-relação. Por outro lado, ressalta-se o ressurgimento, com outros enfoques, das doenças infecciosas entre os determinantes das DCNT (Knobler et al., 2004).

A iniqüidade social é preferencialmente comentada em relação aos países em desenvolvimento, a despeito da sua presença em alguns dos países muito desenvolvidos.

A epidemiologia das DCNT éincompleta e complexa. Nenhum dos fatores de risco para a quase totalidade das DCNT é necessário, suficiente, ou necessário e suficiente (Susser, 1973. Citado por Pereira, 1995). Curiosamente, a grande maioria dos fatores de risco para doenças cardiovasculares são os mesmos para o diabetes, para a doença renal crônica e compartiIhados por uma variedade de neoplasias malignas. A síndrome metabólica, composta por vários elementos ateroscleróticos, está entre os mais comentados determinantes das doenças cardiovasculares e do diabetes na atualidade, com especial relação com a inflamação. Supostamente, a presença de vários desses fatores na epidemiologia de diferentes doenças facilitaria as ações preventivas e de controle além da utilização racional dos recursos humanos e finan- 
ceiros, mas não ocorre na prática ( $M$ cGlynn et al., 2004; M cGlyn, 2004).

A excepcional importância dos fatores comportamentais e ambientais não permite rejeitar o campo biológico, representado pela herança, nem descartar a participação de agentes vivos, como, por exemplo, do papiloma vírus humano em relação ao câncer do colo do útero (Brasil, Ministério da Saúde, 1993). Vários outros microorganismos foram descritos como associados a aterosclerose de modo geral, ao infarto agudo do miocárdio e aos acidentes vasculares encefálicos (Knobler et al., 2004) ou a outros agentes epidêmicos, como o vírus da influenza (Collins, 1932), responsável por grande epidemia no início do século passado, e atribuída por al guns autores a epidemia de doenças cardiovasculares da metade do mesmo século nos países desenvolvidos (Azambuja, 2002).

A expressão clínica das DCNT faz-se após longo tempo de exposição aos fatores de risco e da convivência assintomática do indivíduo com a doença não diagnosticada, mesmo quando os fatores de risco são perceptíveis (tabagismo, obesidade generalizada, obesidade central com ou sem obesidade generalizada, alcoolismo, sedentarismo, etc). Conseqüentemente, os diagnósticos são em fases tardias, com a doença já complicada ou num desfecho que pode ser 0 primeiro e fatal, como ocorre muitas vezes com a doença coronariana aguda e com 0 acidente vascular encefálico. As DCNT correspondem à maior proporção das mortes nos países industrializados e nos emergentes. Para esses últimos as projeções são pessimistas: concentrarão 0 maior número de mortes por DCNT e a maior população mundial com essas doenças em torno do ano 2050.

\section{Vigilância epidemiológica para DCNT}

As doenças cardiovasculares são a primeira causa de morte no mundo. 0 câncer ocupa a segunda ou terceira posição nos países desenvolvidos, mas, como o diabetes, encontra-se entre as 10 primeiras causas de morte em vários países (Yach et al., 2004). Essas mesmas causas são de elevada prevalência bem como seus fatores de risco, muito dos quais considerados doenças ( hipertensão, diabetes, obesidade).

Existem várias propostas de indicadores para VE das DCNT, incluindo mortalidade, morbidade e fatores de risco. Os indicadores gerados pelos países industrializados são por vezes complexos e de difícil aplicação pelos países em desenvolvimento. Países emergentes são inexperientes com a prática da VE para DCNT e para adotá-la são necessárias boas estatísticas de saúde e de dados complementares que impliquem a cobertura populacional, confiabilidade, precisão, qualidade global, abrangência espacial, bem como precisam formar e qualificar recursos humanos, dentre outros pré-requisitos. É importante que os indicadores selecionados estejam de acordo com a realidade do País, caso estes não consigam desenvolver os seus próprios indicadores.

As estatísticas de mortal idade por DCNT são as mais comuns e disponíveis em todo o mundo. A morte, como evento único, exclui o indivíduo da sociedade, devendo ser oficialmente registrada. 0 registro é uma informação de rotina, tornando as estatísticas de mortalidade as menos dispendiosas e de mais fácil obtenção em qualquer país. Por essas razões, as estatísticas de mortalidade são as primeiras consideradas para a VE das DCNT, sendo os coeficientes de mortalidade o primeiro indicador selecionado em todos os países para o acompanhamento da tendência temporal das causas de morte.

Nos países com boas estatísticas hospitalares, sociais, comportamentais, sobre o uso de tecnologias diagnósticas e de tratamentos, econômicas, de incapacidades/invalidez, de programas de prevenção e controle, entre tantos outros, as análises ecológicas de tendência poderão explicar ou sugerir explicações para 0 movimento ascendente ou descendente das taxas de mortalidade e subsidiar decisões para reversão de quadros indesejáveis ou melhoria daqueles cujas tendências já são favoráveis.

Para morbidades, as neoplasias malignas e os eventos cardiovasculares agudos e sintomáticos podem ou poderiam ter suas incidências monitoradas. As demais DCNT são de difícil monitoramento na população pelo longo curso assintomático, por não serem de notificação obrigatória e por serem as coortes populacionais caras e desaconsel háveis para países pobres. Porém, as redes internacionais de programas de prevenção e controle das DCNT são organizadas por regiões, com objetivos e metodologias semelhantes nas regiões, mas com diferenças entre regiões, mostrando ser possível o monitoramento das DCNT em alguns dos países componentes das redes. A maioria deles conta com a participação da Organização 
Mundial da Saúde (OMS) e suas representações nos países dos diversos continentes. Propõem-se que as experiências de sucesso possam ser repassadas a outros países das mesmas regiões, mesmo que não tenham sido planejadas para $\mathrm{VE}$, conquanto o monitoramento de variáveis selecionadas esteja subentendido nas propostas. São exemplos das redes os programas aqui citados por suas siglas: CINDI (Europa), CARMEN (América), NANDI (África), EM AN (Região do M editerrâneo), M OANA (Região do Pacífico), SEARO (Sudeste da Ásia) (WHO, 2001), e ainda o M EGA-COUNTRY, para países com mais de 100 milhões de habitantes dos diversos continentes e o M ONICA, que abrange 32 países com maior concentração na Europa, com milhares de pessoas sob acompanhamento para o estudo das doenças coronarianas (WHO, 1990).

Especificamente nos Estados U nidos, destacam-se os projetos ARIC (White et al., 1996) e o Minnesota H eart Survey (M HS) (M CGovern et al., 1992), iniciados em 1978 e 1970, respectivamente. O ARIC é uma coorte sobre fatores de risco para doenças cardiovasculares e que também atua na vigilância comunitária dos fatores riscos para aterosclerose.

Outra fonte importante de acompanhamento da morbidade, de alerta e de disseminação da informação é o National $\mathrm{H}$ ealth and Nutrition Examination Survey (N HANES), do National Institute of Heart, Blood, and Lung (NIHBL). Esse inquérito vem sendo realizado a cada 10 anos nos Estados Unidos, desde o início da década de 1980 e complementado anualmente com outras informações relacionadas à saúde.

Outras fontes de dados de morbidade são hospitalares e não têm a mesma validade dos dados primários procedentes dos programas ou dos estudos referidos dos quais saem milhares de publicações anuais.

Os investimentos em monitoramento dos fatores de risco para DCNT são os mais importantes. É neste ponto que se quebra a cadeia epidemiológica da doença com todos os benefícios que não se alcançam com a prevenção secundária e menos ainda com a terciária. Contudo, apesar de os fatores de risco mutáveis serem aqueles com as mais fortes e consistentes associações com DCNT, eles são comportamentais, exigindo estratégias de intervenção convincentes, consistentes, inovadoras, sem coerções e muito bem elaboradas para serem bem-sucedidas. Não é tarefa fácil e o maior exemplo de fracasso é a citada epidemia de obesidade, abrangendo faixas etárias precoces.

0 mais conhecido e, provavelmente, o me Ihor emais amplo acompanhamento dos fatores de risco para DCNT, com excelente resposta de participação populacional, éo Behavioral Risk Factor Surveillance Survey System (BRFSS, Estados Unidos, que monitora os fatores de risco para DCNT em todos os estados americanos por inquéritos com conteúdos obrigatórios e, paralelamente, outros de interesse de estados específicos, a depender de outras exposições das suas populações (www.nih.gov).

\section{Brasil: doenças crônicas não-transmissíveis}

Há pelo menos quatro décadas os brasileiros convivem com as doenças cardiovasculares como primeira causa de morte (Lessa, 1998), com o excessivo aumento da mortalidade pelo diabetes nas últimas décadas (Lessa, 2004 - relatório de pesquisa à SVS-M S); ascensão de algumas neoplasias malignas como causa de morte; prevalências elevadas de múltiplos fatores de risco para as DCNT (Lessa et al., 2004) e, sobretudo, com a predominância da medicina curativa. Os programas da hipertensão arterial e diabetes, criados na década de 1980 (Lessa, 1998), não apresentaram o desempenho esperado em razão de mudanças e de substituições ou desativações temporárias por cada nova administração central na área da saúde, sem que os mesmos tivessem sido avaliados. Em outros momentos, os programas não receberam - e ainda não recebem - 0 apoio essencial à sua manutenção e sucesso. Foram programas com propostas de educação e controle direcionados à hipertensão e ao diabetes, mas que não conseguiram se enquadrar na prevenção primária, de baixo custo, desviando-se exclusivamente para a prática clínica, onerosa, de restrito acesso e baixa cobertura.

No contexto epidemiológico e social do Terceiro Mundo, as previsões futuras para o Brasil, em relação às DCNT, até o momento, são sombrias. Persistem as políticas de saúde do País em optar maciçamente pela medicina curativa, pelo atendimento e tratamento das DCNT em serviços de urgência, emergência ou sob hospitalizações. 0 custo elevado dessas opções, obviamente reconhecido, não favorece as mudanças desejáveis. Esse é um aspecto já bastante conhecido daqueles que lidam com 
DCNT, dependente da burocracia, de difícil resolução, impedindo a prática da promoção e proteção da saúde, mesmo que se tenha incorporado essa idéia nos programas oficiais para tais doenças, em períodos governamentais sucessivos desde a década de 1980.

Nas próximas duas a três décadas, os atuais adultos jovens dependentes da assistência do SUS serão a grande massa de idosos que deixaram de receber, no momento adequado, a promoção e proteção para a saúde e/ou controle de DCNT, freqüentemente instalada em idade precoce. Portanto, sem planejamento adequado e metas cumpridas para atender a esses futuros idosos, o envelhecimento ocorrerá com altas prevalências de simultâneas $D C N T$, parte delas com incapacidade. A projeção de pessoas $\geq 60$ anos para o Brasil em 2050 é de $18 \%$ do total, a mesma prevista para o grupo 0-14 anos (IBGE, 2004) e atualmente as DCNT já representam $66 \%$ da carga de doença no País (Schramm et al., 2004). A longevidade cursará sem qualidade de vida e a seguridade social estará mais sobrecarregada, com difícil retorno.

\section{Vigilância epidemiológica no Brasil}

A experiência brasileira com VE para doenças infecciosas e parasitárias está consolidada há várias décadas com sucesso, como no caso da manutenção da eliminação de al gumas doenças infecto contagiosas agudas e a erradicação de outras, todas passíveis de preven ção primária por imunização ativa. Pode-se antever sucesso, embora ainda distante do ideal, para a emergente Aids. M enos evidente ou com retrocessos têm sido os resultados para morbidade por doenças infecciosas de curso prolongado (tuberculose) ou parasitárias endêmicas. Essas últimas são situações em que, vetores ou hospedeiros intermediários participam da cadeia epidemiológica da doença, com forte dependência, por uma lado, das condições sócio-ambientais desfavoráveis e, por outro, da educação para a saúde inexistente ou muito precária. São exemplos: malária, chagas e esquistossomose e a Leishmaniose. 0 sucesso da prevenção, controle ou eliminação, nessas situações, dependeria fortemente de outras áreas sociais, que não a da saúde. Entretanto, sucessos alcançados não permitem falhas na VE, sendo os maiores exemplos do descuido o retorno de doenças graves, não ameaçadoras por muitos anos, mas que resultaram em grandes epidemi- as em passado recente em todo o País (cólera, dengue) ou em focos regionais (febre amarela).

\section{VE em DCNT}

A VE para DCNT foi aventada em 1993, quando gestores do M inistério da Saúde promoveram um amplo seminário incluindo participantes das Secretarias de Saúde da mai oria dos Estados. Os documentos resultantes foram publicados no Informe Epidemiológico do SUS, 1994. Em 2002, foram ministrados cursos especiais sobre a epidemiologia das DCNT no Brasil e incursões sobre a VE para essas doenças patrocinados pelo então Centro Nacional de Epidemiologia/M S (CENEPI/M S). Esses cursos contaram com a participação dos Estados de todas as regiões do País.

Excetuando-se o programa do câncer, existem no País apenas experiências pontuais de VE para DCNT. Desenvolvem-se em pequenas localidades e têm pouco tempo de acompanhamento, com respostas não divulgadas na literatura científica. Um deles, no município de Quirinópolis (GO), é oficialmente o representante brasileiro da rede CARM EN, apoiado parcialmente pela Organização Pan-Americana da Saúde. É uma proposta que se viabiliza pela adesão voluntária de municípios e da população. Em M arília, Estado de São Paulo, também se desenvolve o projeto CARMEN de modo mais independente, ou seja, apoiado pelo município. Alguns Estados têm programas não atuantes e outros estão motivados e/ou empenhados na sua implementação. Embora o tabagismo esteja no rol dos fatores de risco, éo único que se destaca na VE, incluindo-se escolares na faixa etária inicial da adolescência.

Um outro tipo de monitoramento que se destaca no Brasil é o registro do câncer de base populacional, com participação de alguns Estados. Esse registro se refere aos casos de câncer diagnosticados no ano de referência, quando o caso é sintomático ou, mais raramente, quando se trata de detecção precoce. Essa é a maior experiência com VE demorbidade no País, mas mantém-se inconsistente na coleta de dados em quase todos os Estados participantes e a metodologia é sujeita a vieses.

$\mathrm{Na}$ Secretaria de Vigilância em Saúde/M inistério da Saúde, criada em 2003, está em discussão a implementação ampliada da Vigilância Epidemiológica para as DCNT, como uma das prioridades atuais. Abrem-se perspectivas 
de aten ção especial a esses problemas no Brasil, com propostas de estratégias que podem conduzir a bons resultados.

\section{Bases para a VE das D CNT}

As estatísticas de mortalidade das DCNT no Brasil mostram tendência decrescente não explicada para algumas delas (cerebrovasculares e isquêmicas do coração), e ascendente para outras (diabetes e al guns tipos de câncer). Em qualquer dos casos, são de tal magnitude de custos em idades economicamente ativas que requerem estratégias imediatas que contribuam para reduzir ou, pelo menos, limitar os seus avanços. As figuras de 1 a 3 ilustram as tendências à mortalidade para doenças do aparelho circulatório, cerebrovasculares e isquêmicas do coração por 100 mil homens e mulheres, nas regiões brasileiras, no período 19802001; e a figura 4, os coeficientes de mortalidade pelo diabetes por sexo, para o Brasil no mesmo período. A dinâmica da tendência da mortalidade pelo diabetes, estimada de modo simplificado, encontra-se na tabela 1. Tanto os dados brutos quanto os ajustados demonstram excessivo aumento no período.
Esforços devem ser feitos para a redução da morbidade que tende a elevar-se sobretudo diante do envelhecimento populacional. $N$ ão se observa investimento concomitante na promoção da saúde, prevenção primária nem garantia do tratamento contínuo, incluindo-se as complicações que levam à incapacidade invalidez. 0 Brasil é um dos raros países a dispor de estatísticas atual izadas sobre internações por todas as causas e em todo o País, porém, somente para aquelas financiadas pelo SUS, edisponibilizadas pelo Datasus (www.datasus.gov. br), sob a denominação de Sistema de Informação Hospitalar (SIH). Com essa restrição, desconhecem-se as estatísticas referentes a 25\% da população brasileira, que é a parcela assistida por planos e seguros privados de saúde, socialmente melhor inserida.

Por várias razões dependentes do modelo e da prática assistencial, as estatísticas procedentes da rede pública e da conveniada ao SUS são de pouca confiabilidade. Portanto, mesmo com um bom sistema de informação, a VE por estatísticas de altas hospitalares apresenta uma ampla gama de vieses. Exemplos são a possibilidade de múltiplas internações anuais de uma mesma pessoa por uma mesma causa, a dependência do acesso a esse tipo de assistência, os casos mais

\section{Figura 1}

Coeficientes de mortalidade por doenças do aparelho circulatório, ajustados por idade. Regiões brasileiras, 1980-2001.

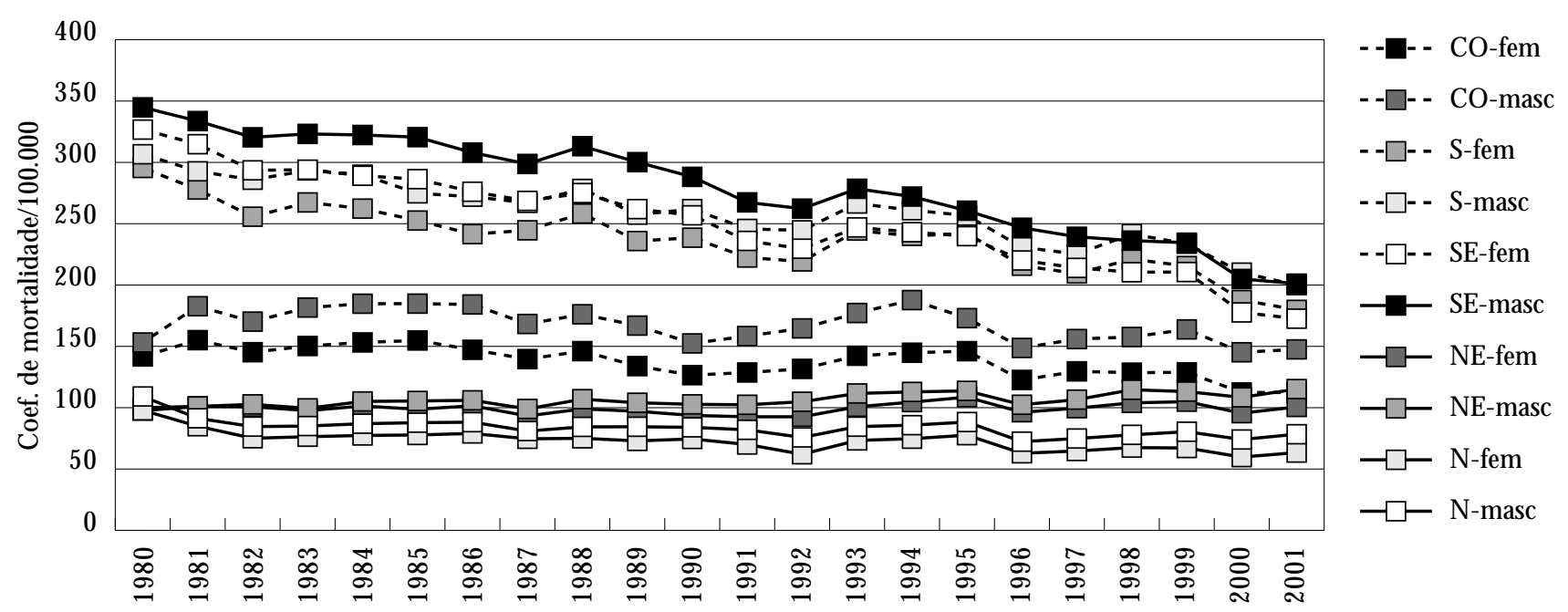




\section{Figura 2}

Coeficientes de mortalidade por doenças cerebrovasculares, ajustados por idade. Brasil, 1980-2001.

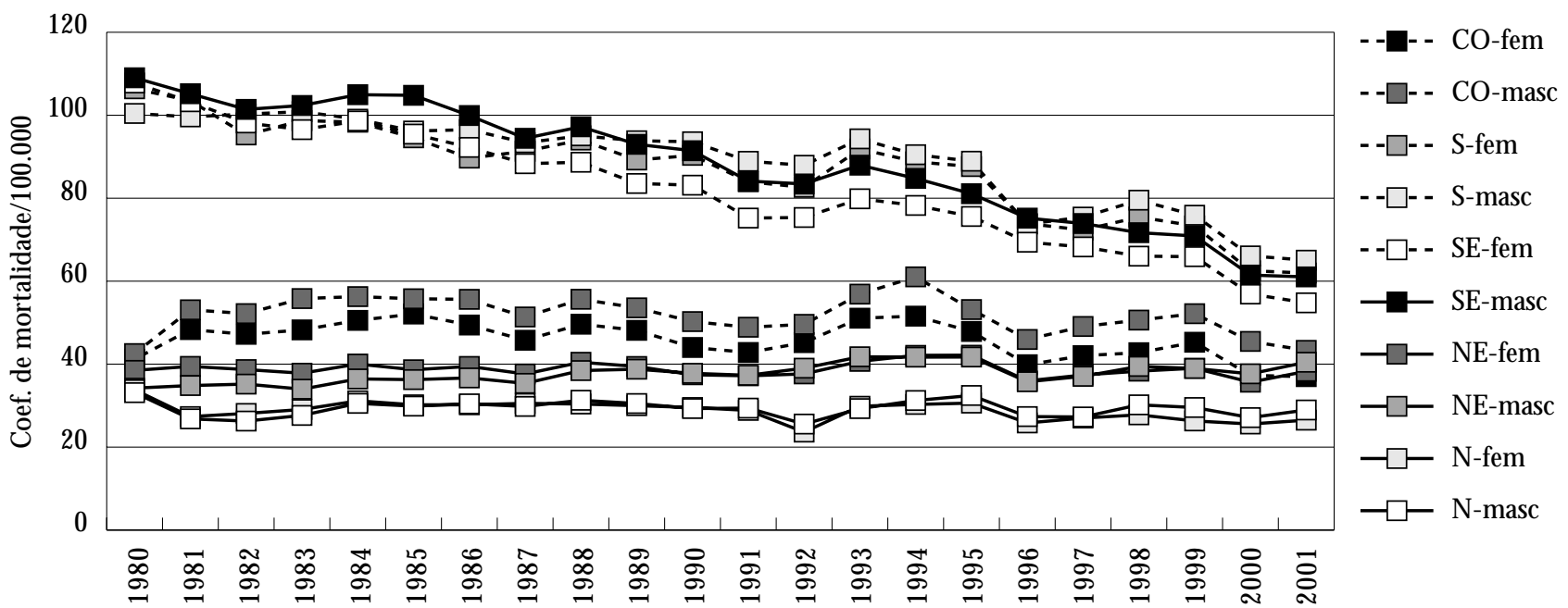

\section{Figura 3}

Coeficientes de mortalidade por doenças isquêmicas do coração, ajustados por idade. Brasil, 1980-2001.

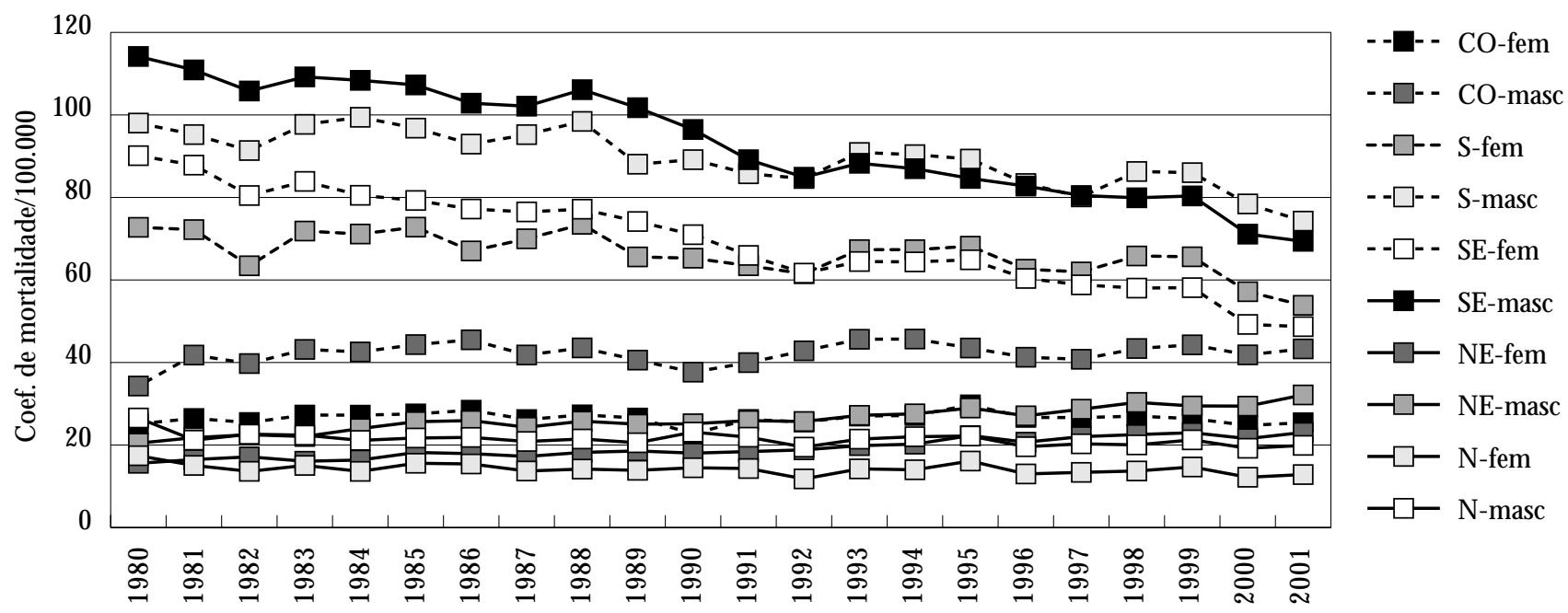




\section{Figura 4}

M ortalidade pelo diabetes mellitus, ajustada por idade. Brasil, 1980-2001.

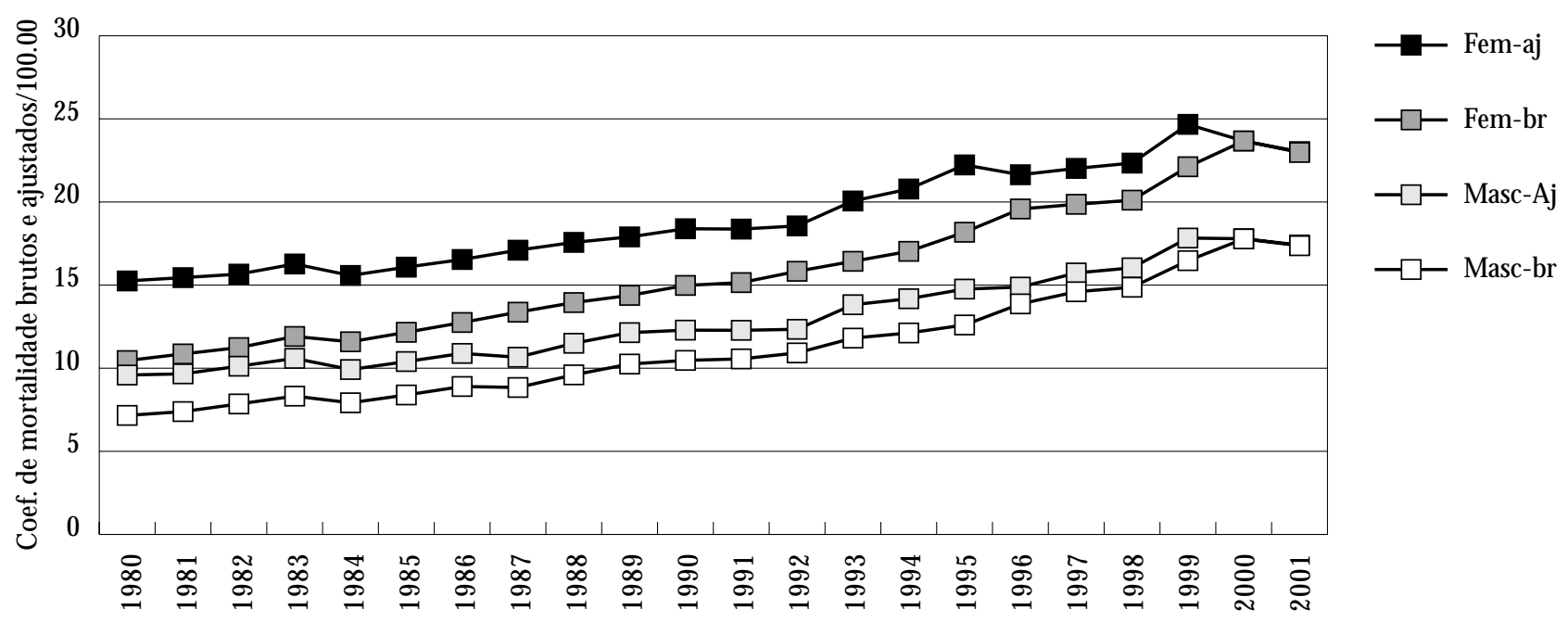

graves serem mais representados nas estatísticas hospitalares; opções por diagnósticos de maior custo e ausência de um bom denominador para indicador de hospital ização. Por outro lado, a mortalidade hospitalar, que poderia ser outro bom indicador, inclusive da qualidade da assistência, é subestimada em razão de o denominador dos cálculos corresponder ao número de hospitalizações em substituição ao número de pessoas internadas pelo evento que determinou a morte. Prestam-se melhor ao monitoramento do tempo médio de permanência hospitalar e do custo médio por internação, mesmo que uma pessoa tenha sido internada pela mesma causa por várias vezes, no período sob enfoque.

Entende-se que são imprescindíveis estratégias que permitam a implementação de sistemas de VE abrangente, mas para isso seria importante algumas observações, correções e ajustes das fontes de informação para os dados que deverão ser utilizados para a VE.

Em princípio é preciso clareza no que se quer vigiar/monitorar. As questões abaixo poderão ajudar nas respostas e decisões.

1) 0 que monitorar?

A seleção dos agravos que se deseja monitorar é de suma importância. Obviamente deverá ser um problema de saúde pública, de grande impacto em populações jovens e em idades economicamente ativas, de elevado custo social, que existam os meios para evitar (promoção da saúde/prevenção primária) ou minimizar a sua ocorrência (tratamento e controle), que esses meios sejam aceitos pela população e acessíveis a todas as classes sociais. É importante observar que em algumas situações a seleção da condição a ser monitorada é de elevada mortalidade, mas de muito baixa prevalência. Exemplo: no Brasil, os acidentes vasculares cerebrais (encefálicos) são a primeira causa de morte. Sua prevalência é muito baixa, a letalidade é el evada e as seqüe las são computadas como incapacidade ou invalidez, porém os seus principais fatores de risco são de alta prevalência - a hipertensão arterial e 0 diabetes. 0 impacto social é grande.

2) Onde monitorar?

A condição tem a mesma importância em todo o País? A decisão deve ser racional. As prioridades podem diferir entre as regiões. No caso das DCNT, se uma condição não é prioridade atual poderá vir a ser em futuro próximo, caso a vigilância não se faça sobre os seus fatores de risco. Nesse caso os benefícios com monitoramento dos fatores de risco se configuram como ideal.

3) Como monitorar?

Definida a condição a vigiar, é preciso conhecer como ela se apresenta como problema maior de saúde desde as freqüências dos seus fatores de risco ao seu impacto social. 
Tabela 1

Coeficientes de mortalidade pelo diabetes e cálculo simplificado da tendência no Brasil, período 1980-2001.

\begin{tabular}{lcccc}
\hline & \multicolumn{2}{c}{ Sexo masculino } & \multicolumn{2}{c}{ Sexo feminino } \\
Período & Coeficiente 100.000 & Tendência\% & Coeficiente100.000 & Tendência\% \\
\hline 1980 & 7,16 & & 10,46 & \\
1981 & 7,39 & 3,1 & 10,86 & 3,7 \\
1982 & 7,85 & 5,9 & 11,24 & 5,4 \\
1983 & 8,32 & 5,6 & 11,91 & $-2,8$ \\
1984 & 7,92 & $-5,1$ & 11,59 & 4,7 \\
1985 & 8,39 & 5,6 & 12,16 & 4,6 \\
1986 & 8,9 & 5,7 & 12,75 & 4,6 \\
1987 & 8,84 & $-0,7$ & 13,37 & 4,2 \\
1988 & 7,9 & 13,95 & 3,0 \\
1989 & 9,6 & 6,3 & 14,38 & 4,0 \\
1990 & 10,25 & 2,1 & 14,98 & 1,1 \\
1991 & 10,47 & 0,9 & 15,15 & 4,3 \\
1992 & 10,56 & 3,3 & 15,83 & 3,7 \\
1993 & 10,92 & 7,5 & 16,43 & 3,5 \\
1994 & 11,81 & 2,6 & 17,03 & 6,3 \\
1995 & 12,12 & 3,8 & 18,18 & 7,2 \\
1996 & 12,6 & 19,58 & 1,4 \\
1997 & 13,87 & 9,2 & 19,86 & 1,2 \\
1998 & 14,6 & 5,0 & 20,11 & 9,1 \\
1999 & 14,87 & 1,8 & 22,12 & 6,5 \\
2000 & 16,47 & 9,7 & 23,67 & $-3,0$ \\
2001 & 17,78 & 7,4 & 22,98 & 76,4 \\
\hline Incremento no período & 17,37 & $-2,4$ & &
\end{tabular}

Conforme a resposta, identificam-se a disponibilidade de fontes de informação, a existência de bases de dados para cada uma delas e a sua confiabilidade para o monitoramento. Pode-se monitorar com base exclusiva em dados secundários, primários ou ambos.

0 monitoramento baseado em dados secundários é economicamente vantajoso, mas as informações dos bancos de dados devem ser confiáveis. A mortalidade só pode ser monitorada por dados secundários, excetuando-se em estudos especiais, como as coortes, mas inadequadas para VE. Antes de uma decisão sobre o monitoramento da mortalidade, seria importante a validação de alguns diagnósticos registrados nos certificados de óbito. Em países como o Brasil, uma grande parcela dos óbitos ocorre sem assistência médica prévia e sem 0 diagnóstico confirmado. Muitas das mortes com sintomatologia de curta duração podem ter diagnósticos por conveniências ou por pressupostos. Exemplo é o infarto agudo do miocárdio. Muitos óbitos declarados como hospitalares, de fato não o foram. Essas são apenas algumas considerações sobre distorções de diagnósticos da causa da morte.

Bancos de dados secundários para morbidade quase nunca têm representatividade populacional, mas existem exceções: as neoplasias malignas com curto curso clínico são um exemplo. Entre os cuidados com a informação, devese também estar atento para não duplicar ou triplicar um mesmo caso, quando se usam várias fontes de diagnóstico e/ou tratamento.

0 uso de dados primários é o ideal. Os inquéritos têm a capacidade de produzir um conjunto de informações diretamente obtidos da população. A amostragem, a metodologia e a padronização de técnicas e de entrevistadores são aspectos importantes nesse tipo de informação bem como a análise de consistência das informações e o controle de qualidade. São mais difíceis de execução se a cobertura for para um país das dimensões do Brasil. São mais caros do que a coleta de dados secundários, porém mais compensadores. Se programados para periodicidade compatível com os recursos disponíveis, em prazos não superiores a 10 anos, os custos 
se diluem. Além do mais, podem ser intercalados com mini-inquéritos específicos, como se faz nos Estados Unidos.

4) $O$ que medir?

A questão se refere à seleção dos indicadores. Em países sem experiência com DCNT devem-se selecionar cuidadosamente um mínimo de indicadores que sejam capazes de retratar o que se pretende com a menor possibilidade de erro possível. 0 número de indicadores deve ser o suficiente para acompanhar mudanças da condição sob monitoramento e indicar o que deve ser corrigido com intervenções, ou se 0 indicador é adequado para descrever ou analisar tendências. Quanto maior o número de indicadores selecionados por países inexperientes, tanto maiores as possibilidades de: a) fracasso com a metodologia; b) interrupção do processo de vigilância; c) necessidade de maior contingente de recursos humanos qualificado para análises e interpretações; d) não interpretação dos resultados; e) não avaliação das intervenções.

0 tipo de análise programado deve ser simples, como recomendado por alguns autores (Luepker et al., 2004). Deve ser padronizado para o País, usando ajustamentos por idade e sexo para comparações. No Brasil, normalmente não existem pesquisadores nos órgãos governamentais responsáveis pela saúde pública, onde se centralizam os bancos de dados e outras informações, e parece inviável preparar equipes com esse perfil para todo o País. É mais razoável uma análise mais simples, sem recursos tecnológicos avançados, mas que possa ser descentralizada e executada sem interrupções e capaz de oferecer respostas aos problemas na velocidade desejada pelos planejadores e gestores dos programas de saúde. Análises de tendência temporal por metodologias sofisticadas, por modelos de regressão, que podem variar de uma para outra causa sob vigilância, devem ser evitadas, deixando-as para investigadores das academias. Talvez, em tempo futuro seja possível pensar num centro especial para concentração das análises mais complexas para todo 0 País. Para isso é preciso que os programas estejam consolidados na prática e que haja domínio da prática VE.

Desde 2002 e pela primeira vez, a OM S vem recomendando aos diversos países a implementação da vigilância para DCNT, com enfoque nos maiores fatores de risco que predizem as mais comuns delas (Armstrong \& Bonita, 2003). Essa proposta é o "STEPwise approach to non-communicable disease" ( $M$ étodo Pro- gressivo) e vem sendo apresentada aos países em fórum itinerante, sempre patrocinada pela OM S e suas representações. 0 III Forum realizou-se no Brasil em novembro de 2003, em parceria com a Secretaria de Vigilância em Saúde/M inistério da Saúde. Parece ser o método mais racional, especialmente para os países emergentes. 0 enfoque sobre os fatores de ris$\mathrm{co}$, como organizado, pode ser trabalhado por etapas, o que caracteriza a progressividade da proposta. O M étodo consta de três passos, usando dados do passado, presente e futuro. Para investigação dos fatores de risco, cada passo contém componentes principal, ampliado e opcional. N os quadros 1 e 2 aparecem sumarizados os conteúdos propostos no método; a figura correspondente é elucidativa e pode ser observada no documento original da OMS.

0 tempo passado do quadro 1 se refere a óbitos nos três passos. 0 indicador usado é a taxa de mortal idade específica por idade, sexo e causa, dependendo do passo (dados secundários). A penas no passo 2 a autópsia verbal é obtida por entrevista (dados primários) e 0 indicador não é taxa de mortalidade. 0 tempo presente refere-se a dados de morbidade, sendo baseada em dados secundários nos passos 1 e 2 e em dados primários (prevalência e incidência) no passo 3. No tempo futuro os dados são primários (inquéritos) e os três passos estão direcionados para fatores de risco " referidos" (tabagismo, sedentarismo, etc.), medidos (antropométricas e da pressão arterial) ou dosados (bioquímica).

0 quadro 2 é exclusivo para fatores de risco. 0 módulo principal é simplificado nos três passos. Busca o essencial no inquérito (passo 1), medidas antropométricas básicas no passo 2, e bioquímica básica no 3.

0 modo ampliado é um aprofundamento do principal e no conteúdo do optativo, as variáveis a serem questionadas dependerão da necessidade e dos recursos alocados ao programa, embora seja desejável a sua realização. 0 detalhamento deste método, incluindo descrição dos fatores de risco, encontra-se na referência original.

Observando o modelo, o Brasil dispõe das informações sobre óbitos nos três passos referentes aos óbitos (passado), exceto a autópsia verbal, só disponível nesse momento para o infarto agudo do miocárdio em cinco capitais (Belém, M aceió, Salvador, Vitória e Cuiabá), onde na validação da declaração de óbito foram elevadas as proporções de diagnósticos fal- 
Quadro 1

M étodo progressivo da OMS para vigilância das DCNT.

\begin{tabular}{llll}
\hline $\begin{array}{l}\text { DCNT } \\
\text { Óbitos } \\
\text { (passado) }\end{array}$ & $\begin{array}{l}\text { Passo 1 } \\
\text { Taxas de mortalidade } \\
\text { por idade e sexo }\end{array}$ & $\begin{array}{l}\text { Passo } 2 \\
\text { Taxas de mortalidade por } \\
\text { idade, sexo e causa de } \\
\text { morte (autópsia verbal) }\end{array}$ & $\begin{array}{l}\text { Passo 3 } \\
\text { Taxas de mortalidade por } \\
\text { idade, sexo e causa de morte } \\
\text { (certificado de óbito) }\end{array}$ \\
\hline $\begin{array}{l}\text { Doenças } \\
\text { (presente) }\end{array}$ & $\begin{array}{l}\text { Admissões em clínicas / } \\
\text { hospitais por idade e sexo }\end{array}$ & $\begin{array}{l}\text { Taxas e doença principal } \\
\text { em 3 grupos: doenças } \\
\text { transmissíveis, DCNT } \\
\text { elesões }\end{array}$ & $\begin{array}{l}\text { Incidência ou prevalência } \\
\text { da doença pela causa } \\
\text { concreta }\end{array}$ \\
\hline $\begin{array}{l}\text { Fatores de risco } \\
\text { (futuro) }\end{array}$ & $\begin{array}{l}\text { Informações baseadas } \\
\text { em inquérito sobre } \\
\text { fatores de risco chave }\end{array}$ & $\begin{array}{l}\text { Inquérito + medidas } \\
\text { antropométricas }\end{array}$ & $\begin{array}{l}\text { Inquérito + medidas } \\
\text { antropométricas + exames } \\
\text { bioquímicos }\end{array}$ \\
\hline
\end{tabular}

Reprodução autorizada pela OM S para fins não-comerciais. WHO 2001. Surveillance of risk factors for non Communicable disease. The WHO STEPwise approach. WHO, Geneva. Traduzido para o português pela autora.

Quadro 2

M étodo progressivo da OM S para avaliação de fatores de risco.

\begin{tabular}{llll}
\hline Módulos & $\begin{array}{l}\text { Passo 1: } \\
\text { Questionário }\end{array}$ & $\begin{array}{l}\text { Passo 2: Medidas } \\
\text { antropométricas }\end{array}$ & $\begin{array}{l}\text { Passo 3: Medidas } \\
\text { bioquímicas }\end{array}$ \\
\hline Principal & $\begin{array}{l}\text { Variáveis socioeconô- } \\
\text { micas e demográficas, } \\
\text { consumo de cigarro } \\
\text { e deálcool, inatividade } \\
\text { física, nutrição }\end{array}$ & $\begin{array}{l}\text { Peso, altura, } \\
\text { circunferência da } \\
\text { cintura, pressão arterial }\end{array}$ & $\begin{array}{l}\text { Glicemia de jejum e } \\
\text { colesterol total }\end{array}$ \\
\hline Ampliado & $\begin{array}{l}\text { Modelos de alimentação, } \\
\text { educação e indicadores } \\
\text { familiares }\end{array}$ & $\begin{array}{l}\text { Circunferência } \\
\text { dos quadris }\end{array}$ & $\begin{array}{l}\text { HDL-colesterol, } \\
\text { triglicérides }\end{array}$ \\
\hline $\begin{array}{l}\text { Optativo } \\
\text { (exemplos) }\end{array}$ & $\begin{array}{l}\text { Outros comportamentos } \\
\text { relacionados com a saúde, } \\
\text { saúde mental, } \\
\text { incapacidades e lesões }\end{array}$ & $\begin{array}{l}\text { Caminhada cronometrada, } \\
\text { pedômetro, espessura } \\
\text { de pregas cutâneas, } \\
\text { freqüência do pulso }\end{array}$ & $\begin{array}{l}\text { Teste de tolerância à } \\
\text { glicose, exame de urina }\end{array}$ \\
\hline
\end{tabular}

Reprodução autorizada pela OM S para fins não não-comerciais. WHO 2001. Surveillance of risk factors for

non Communicable disease. TheWHO STEPwise approach. WHO, Geneva. Traduzido para o português pela autora.

sos positivos ( Lessa et al., 2004. Relatório de pesquisa à SVS-MS). Esse procedimento énecessário para al gumas causas de morte, em particular as mortes súbitas ou eventos agudos com poucas horas de duração, especial mente quando o óbito ocorre sem assistência médica e fora do hospital, sem que a autópsia tenha sido solicitada. Para o tempo presente, passos 1 e 2, dispomos das estatísticas hospitalares do SUS, com as críticas já referidas. No passo 3 (futuro) os estudos multicêntricos para o diabetes, estado nutricional e tabagismo estão desatualizados, embora se disponha de informações pon- tuais em áreas do País, sobre essas condições, mas sem programação para vigilância e sem padronização metodológica.

Estão em fase de análise as informações do inquérito sobre fatores de risco referidos para DCNT, desenvolvido em 16 capitais entre 2002 e 2004, que poderiam ser o início da vigilância dos fatores de risco, pois preenchem os conteúdos descritos para o passo 1 dos três módulos do quadro 2. A restrição é que os dados não re presentam o País, mas é uma etapa cumprida para as capitais participantes do inquérito. Para os demais passos existem informações pontu- 
ais de boa e de má qualidade, metodologicamente diferentes e não planejados para vigilância, sem abrangência e portanto, não podem ser usadas para essa finalidade.

Para a implementação da VE para DCNT, usando o método progressivo, são necessárias três decisões técnicas importantes quanto aos dados de óbito: a primeira é decidir se a qualidade dos dados procedentes de vários Estados especialmente do Nortee de al guns do Nordeste se prestam, nesse momento, ao monitoramento; a segunda é validar a causa da morte registrada nos certificados de óbito por autópsia verbal, em várias localidades do País, de modo que se possa corrigir distorções; a terceira éa definição parcimoniosa dos indicadores para mortalidade.

Para o uso dos dados de morbidade hospitalar - internações e taxa de mortalidade hospitalar - , será necessário refletir sobre a sua confiabilidade e definir os indicadores. É preciso também tentar recuperar informações dos planos e seguros de saúde, uma vez que a vigilância baseada exclusivamente no SIH seria parcial e distorcida, procedente das classes sociais mais pobres, não refletindo a realidadenacional.

Para a parte mais importante da VE, o módulo dos fatores de risco, os inquéritos progra- mados são viáveis. A questão maior parece ser a adequação de recursos para que esses inquéritos venham a ser prioridade real para as DCNT no País. Essa decisão, no entanto, só será pertinente se o setor responsável pela atenção básica à saúde incorporar, de fato e de direito, a prática da promoção da saúde, da prevenção primária, visando à eliminação ou minimização dos fatores de risco para DCNT na sociedade. Se isso não ocorrer, a VE será desnecessária.

A VE para DCNT no Brasil é uma decisão que não pode retroceder, mas será necessário um grande esforço para participação e colaboração de todos os estados, sem interrupções. 0 método progressivo da OM S é uma proposta válida, coerente, e poderá ser implementada selecionando-se as etapas com disponibilidade de dados, facilitando a adaptação dos recursos humanos a esse tipo de vigilância, especialmente nos Estados com maiores dificuldades com a organização da informação e análise. 0 ideal seria incluir de imediato a VE dos fatores de risco em todo o País. Com alguns ajustes nas fontes de dados, com a prática da prevenção precoce e garantindo-se a coleta de dados primários em períodos pré-definidos, pode-se afirmar que a VE para DCNT éviável.

\section{Referências bibliográficas}

Armstrong T \& Bonita R 2003. Capacity building for an integrated non-communicable disease risk factor surveillance system in developing countries. Ethnicity and Disease 13(Suppl 2):S13-18.

Azambuja MI \& Duncan BB 2002. Similarities in patterns of mortality from influenza $A$ ( $H 1 N 1$ strains) in the first half of the 20th century and the rise and fall of coronary heart disease: a new hypothesis concerning the CHD epidemic. Cadernos de Saúde Pública 3:557577.

Beaghole R \& Yach D 2003. Globalization and the prevention and control of non-communicable disease. The neglected chronic disease of adults. Lancet 362:903908.

Collins SD 1932. Excess mortality from causes other than influenza and pneumonia during influenza epidemics. Public Health Report 47:2159-2179.

Desai J et al. 2003. Public health surveillance of diabetes in the United States. Journal of Public H ealth $M$ anage ment and Practice 9(6S):S44-S51. 
Eyre H, Kahn R \& Robertson RM 2004. Preventing cancer, cardiovascular disease, and diabetes. A common agenda for the American Cancer Society, the American Diabetes Association, and the American Heart Association. Diabetes Care 27(7):1812-1824.

Grundy SM 2003. Inflamation, metabolic syndrome and diet responsiveness. Circulation 108:126-128.

IBGE 2004. Ver <http://www.ibge.gov.presidencia/noticia impressao.php?id noticia $=20>$

Jacoby E 2004. The obesity epidemic in the Americas: making healthy choices the easiest choices. Revista Panamericana de Salud Pública 15(4):278-284.

Knobler SL, O'Connor S, Lenon SM \& Najafi M 2004. The infectious etiology of chronic diseases. D efining the re lationship, enhancing the research and mitting the effects. The National Academics Press.

Leeder S, Raymond S \& Greenberg H 2004. A race against time. The challenge of cardiovascular disease in developing economies. The Earth Insitute, Columbia University, the University of Sydney, Columbia University M ailman School of Public Health, Trustees of Columbia University, City of New York, 95 pp.

Lessa I 1998. Os programas nacionais de educação e controle para DCNT, pp. 241-250. In I Lessa (org.). Adulto brasileiro e as doenças da modernidade. Epidemiologia das doenças crônicas não-transmissíveis no Brasil. Hucitec, São Paulo.

Lessa I 1999. Perfil das doenças cardiovasculares no Brasil, pp. 15-30. In D M ion \& F Nobre (orgs.). Risco cardiovascular global. Lemos Editora \& Gráficos Ltda, São Paulo.

Lessa I et al. 2004. Simultaneidade de fatores de risco cardiovascular modificáveis na população adulta de Salvador-Brasil. Revista Panamericana de Salud Pública 16:131-137.

Luepker RV, Evans A, M cKigue P \& Reddy KS 2004. Cardiovascular survey methods. (3a ed.) WHO, Geneva.

M agnus P \& Beaghole R 2001. The real contribution of the major risk factors to the coronary epidemics: time to end "only 50\%" myth. Archives of Internal M edicine 161:2657-2660.

M ayor S 2004. Cardiovascular disease threatens developing countries. Britsh M edical Journal 328:1032.

McGlynn EA 2004. There is no perfect health system. Health affairs. The Police Journal of the H ealth Sphere 23(3):100-102

McGovern PG et al. 1992. Trends in survival of hospitalized myocardial infarction patients mortality, morbidity, and risk factor levels for stroke between 19701985: the Minnesota Heart Survey. Circulation 85:172-179.

M itka M 2004. Heart disease: a global health threat. Journal of the American M edical Association 291:2533.

M oakdad AH, Bowman BA, Ford ES \& Vinicor F 2001. The continuing epidemics of obesity and diabetes in the U.S. Journal American M edical Association 286: 1195-1200.
Murray CJL \& Lopez AD 1996. The global burden of disease. Word Health Organization, Geneva.

Nissinen A, Berrios X \& Puska P 2001.Community-based non-communicable disease interventions: lessons from developed countries for developing ones. Bulletin World Health Organization 29(10):963-970.

Pan-American Health Organization/WHO 2002. CAR$M E N$ - An initiative for integrated prevention of noncommunicable disease in the Americas. PAHO/WHO.

Pereira M G 1995. Epidemiologia - teoria e prática. Editora Guanabara-Koogan, Rio de Janeiro.

Reddy KS \& Yusuf S 1998. Emerging epidemic of cardiovascular disease in developing countries. Circulation 97: 596-601.

Robine JM , Jagger C, EU RO-REVES group 2003. Creating a coherent set of indications to monitor health across Europe: The Euro-Reves 2. European Journal of Public Health 13(3 Suppl):6-14

Schramm JM A et al. 2004. Transição epidemiológica e o estudo de carga de doença no Brasil. Ciência \& Saúde Coletiva 9(4):897-908.

Stein C \& Colditz GA 2004. The epidemic of obesity. Clinicam Endocrinology and M etabolism 89(6):23222525.

White AD et al. 1996. The community surveillance of coronary heart disease in Atherosclerosis Risk in Communitties (ARIC) study: methods and initial two years experience. Journal of Clinical Epidemiology 49:223-233.

World Health Organization 2001. Surveillance of risk factors for non-communicable disease. The WHO STEPwise approach. WHO, Geneva.

World Health Organization 1990. M ONICA project: manual of operations. WHO, Geneva.

World Health Organization 2002. II Global Forum on N on- communicable Diseases Prevention and Control, convened in Shangai, China 2002. WH O, Geneva.

World $\mathrm{H}$ ealth Organization 2003. III Global Forum on Non-communicable Diseases Prevention and Control. Rio de Janeiro, Brazil, 7-14 nov.

World Health Organization 2003. Global Forum on NDC Prevention and Control. Rotating International Visitors program. Report 5 - 9 oct 2003, Isfaham, Islamic Republic of Iran.

Yatch D, Hawkes C, Gould CL \& H offman KJ 2004. Global burden of chronic diseases. Overcoming impediments to prevention and control. Journal of the American M edical Association 291:2616-2622.

Yusuf S, Reddy S, O unpuu S\& Anand S 2001. Global burden of cardiovascular diseases: part 1: general considerations, the epidemiologic transition risk factors, and impact of urbanization. Circulation 104:27462753.

Artigo apresentado em 20/8/2004

Aprovado em 18/9/2004

Versão final apresentada em 15/10/2004 